OPEN ACCESS

Edited by:

Mark Girgis,

University of California, Los Angeles, United States

Reviewed by:

Sivesh Kamarajah,

University Hospitals Birmingham NHS

Foundation Trust,

United Kingdom

Eliza Wright Beal,

The Ohio State University,

United States

*Correspondence:

Tao Li

litao7706@163.com

${ }^{\dagger}$ These authors have contributed equally to this work and

share first authorship

Specialty section:

This article was submitted to

Surgical Oncology,

a section of the journal

Frontiers in Oncology

Received: 07 January 2021

Accepted: 15 April 2021

Published: 03 May 2021

Citation:

Chen Z, Yu B, Bai J, Li Q, Xu B,

Dong Z, Zhi $X$ and LiT (2021)

The Impact of Intraoperative Frozen Section on Resection Margin

Status and Survival of Patients

Underwent Pancreatoduodenectomy

for Distal Cholangiocarcinoma.

Front. Oncol. 11:650585.

doi: 10.3389/fonc.2021.650585

\section{The Impact of Intraoperative Frozen Section on Resection Margin Status and Survival of Patients Underwent Pancreatoduodenectomy for Distal Cholangiocarcinoma}

\author{
Zhiqiang Chen ${ }^{1 \dagger}$, Bingran $\mathrm{Yu}^{1 \dagger}$, Jiaping $\mathrm{Bai}^{2}$, Qiong $\mathrm{Li}^{1}$, Bowen $\mathrm{Xu}^{1}$, Zhaoru Dong ${ }^{1}$, \\ Xuting $\mathrm{Zhi}^{1}$ and Tao $\mathrm{Li}^{1 *}$ \\ ${ }^{1}$ Department of Hepatobiliary Surgery, General Surgery, Qilu Hospital, Cheeloo College of Medicine, Shandong University, \\ Jinan, China, ${ }^{2}$ Qilu Hospital, Cheeloo College of Medicine, Shandong University, Jinan, China
}

Background: Intraoperative frozen section (FS) is broadly used during pancreaticoduodenectomy (PD) to ensure a negative margin status, but its survival benefits on obtaining a secondary $\mathrm{R} 0$ resection for distal cholangiocarcinoma (dCCA) is controversial and unclear.

Methods: Clinical data of 107 patients who underwent PD for dCCA was retrospectively collected and divided into different groups based on use of FS (FS and non-FS groups) and status of resection margin (pRO, sR0 and R1 groups), and clinical parameters and survival of patients were compared and analyzed accordingly.

Results: There were 50 patients in FS group with a median survival of 28 months, 57 patients in non-FS group with a median survival of 27 months. There was no statistical difference between the two groups with Kaplan-Meier survival analysis $(P=0.347)$. There were 98 patients in R0 group (88 in pR0 and 10 in sR0) and nine patients in R1 group, with a median survival of 29 months and 22 months respectively, which showed a better survival in $\mathrm{R} 0$ group than in $\mathrm{R} 1$ group $(P=0.006)$. Survival analyses between subgroups revealed difference between $\mathrm{pR} 0$ and $\mathrm{R} 1$ group $(P=0.005)$, while no statistical difference concerning pRO vs. sR0 $(P=0.211)$ and sR0 vs. R1 groups $(P=0.262)$. Multivariate Cox regression analysis revealed resection margin status, pre-operative biliary drainage and lymph node invasion to be independent prognostic factors for dCCA patients.

Conclusions: Intraoperative FS should be recommended as it significantly increased the rate of $\mathrm{RO}$ resection, which was positively related to a better survival. A primary $\mathrm{RO}$ resection should also be encouraged and if not, a secondary $\mathrm{RO}$ could be considered at the discretion of surgeons as it showed similar survival with primary RO resection.

Keywords: pancreatoduodenectomy, resection margin, survival analysis, frozen section, distal cholangiocarcinoma 


\section{INTRODUCTION}

Cholangiocarcinoma (CCA), a cancer arising from epithelium of biliary tract, is the most common malignancy in biliary duct system and the second common primary liver malignancy in the whole hepatobiliary system after hepatocellular carcinoma (HCC), accounting for about $3 \%$ of all gastrointestinal tumors and $10 \%$ to $15 \%$ of hepatobiliary malignancies $(1,2)$. According to the updated $3^{\text {rd }}$ edition of International Classification Diseases for Oncology (ICD-O) system, CCA are categorized into intrahepatic CCA (iCCA), perihilar CCA (pCCA) and distal CCA (dCCA), accounting for about $5 \%$ to $10 \%, 60 \%$ to $70 \%$, and $20 \%$ to $30 \%$ of all CCA cases, respectively $(2,3)$. Given their differences in frequency, pathobiology, management and prognosis, iCCA, pCCA and dCCA should be viewed as separate entities, and surgery is the only curative treatment for a long-term survival (4). Compared to its two counterparts, dCCA is usually indicated for pancreaticoduodenectomy (PD) or pylorus-preserving pancreaticoduodenectomy (PPPD) with lymphadenectomy which has a higher resectability rate than iCCA or pCCA (5). However, the overall survival of dCCA patients after curative resection was still dismal, with a 5-year survival rate about $18 \%$ to $43 \%(5-7)$.

Resection margin status is considered to be a major prognostic factor of survival for dCCA patients, thus a R0 resection is always pursued by surgeons (7-9). And the only method of assessing intraoperative resection margin status was frozen section (FS) $(10,11)$, but the clinical value of FS on assessing bile duct resection margin is controversial and debated because of its inherent pitfalls and probable disagreement with permanent section (PS) $(12,13)$. In this study we retrospectively analyzed survival outcomes in patients with dCCA who underwent PD for curative resection, aiming to investigate if the use of intraoperative FS could provide survival benefits by obtaining a secondary R0 resection margin. Furthermore, independent prognostic factors of dCCA were also investigated via multivariable Cox regression analysis.

\section{METHODS}

\section{Study Design and Participants}

A retrospective analysis was conducted on patients who underwent PD for dCCA in the General Surgery Department of Qilu Hospital (Cheeloo College of Medicine, Shandong University) from January 2011 to November 2019. dCCA was defined as carcinoma arising from distal part of extrahepatic bile duct that was below insertion of cystic duct. Only patients with pathologically confirmed dCCA that underwent curative PD were included; patients undergoing PD for diseases other than dCCA (such as pancreatic cancer, duodenal cancer, ampullary cancer and benign lesions) were excluded.

All relative clinical data were collected including baseline demographics, tumor characteristics, as well as long-term followup for patients survival. Primary analysis was performed between FS and non-FS groups in order to view if the use of FS had some impact on patient overall survival. Then, a further statistical analysis comparing different margin status were performed to see if the use of FS on obtaining a sR0 could improve long-term survival of patients. Thus, patients were divided into pR0, sR0 and R1 groups accordingly, and clinical parameters and survival of patients were compared and analyzed among groups.

R0 was defined as absence of macroscopic and microscopic tumor cells at the bile duct margin, with two subgroups of pR0 (primary R0) and sR0 (secondary R0, a negative margin achieved by extended resection). R1 was defined as presence of microscopic tumor cells at resection margin, while $\mathrm{R} 2$ resection was defined as macroscopically visualized tumor at margins. Clavien-Dindo classification was used to analyze the postoperative complication of patients (14). Tumor staging was classified according to the 8th edition of TNM classification system from American Joint Committee on Cancer (AJCC) (15).

\section{Statistical Analysis}

The numerical data were expressed as mean \pm standard deviation and assessed by using the Student's $t$-test. The categorical data were presented as percentage and assessed by the chi-square test. Analysis of variance (ANOVA) was used to compare the data among three groups. Postoperative survival was described by Kaplan-Meier curves and comparison between groups was performed using log-rank test. The Cox proportional hazard model was used for multivariable analysis. A $P$ value of less than 0.05 was considered statistically significant. Analyses were performed with SPSS 25.0 (SPSS, Chicago, IL, USA).

\section{RESULTS}

\section{Patient Demographics}

The patient selection and demographics were summarized and shown in Figure 1 and Table 1. Briefly, there were 107 patients with a mean age of 62.3 years and a male to female ratio of 70:37. There were 50 patients in FS group and 57 patients in non-FS group; while there were 98 patients in R0 group ( $88 \mathrm{pR} 0$ plus 10 sR0) and nine patients in R1 group. Preoperative biliary drainage was performed in 71 patients via percutaneous transhepatic cholangial drainage (PTCD, 64 cases), endoscopic nasobiliary drainage (ENBD, three cases) and endoscopic retrograde biliary stent (ERBS, four cases), respectively. Only 33 (30.8\%) patients received post-operative chemotherapy and/or radiotherapy.

\section{Clinical Outcomes Between FS Group and Non-FS Group}

There were 50 patients in FS group and 57 patients in non-FS group. As shown in Table 1, there was no statistical difference between the two groups concerning baseline demographics and tumor characteristics $(P>0.05)$. There was no false negative report for FS in our study, as all the R0 resection margin in FS group was confirmed by postoperative PS. While nine patients in non-FS group were revealed to be $\mathrm{R} 1$ resection that was confirmed by postoperative PS, making the rate of $\mathrm{R} 1$ resection 


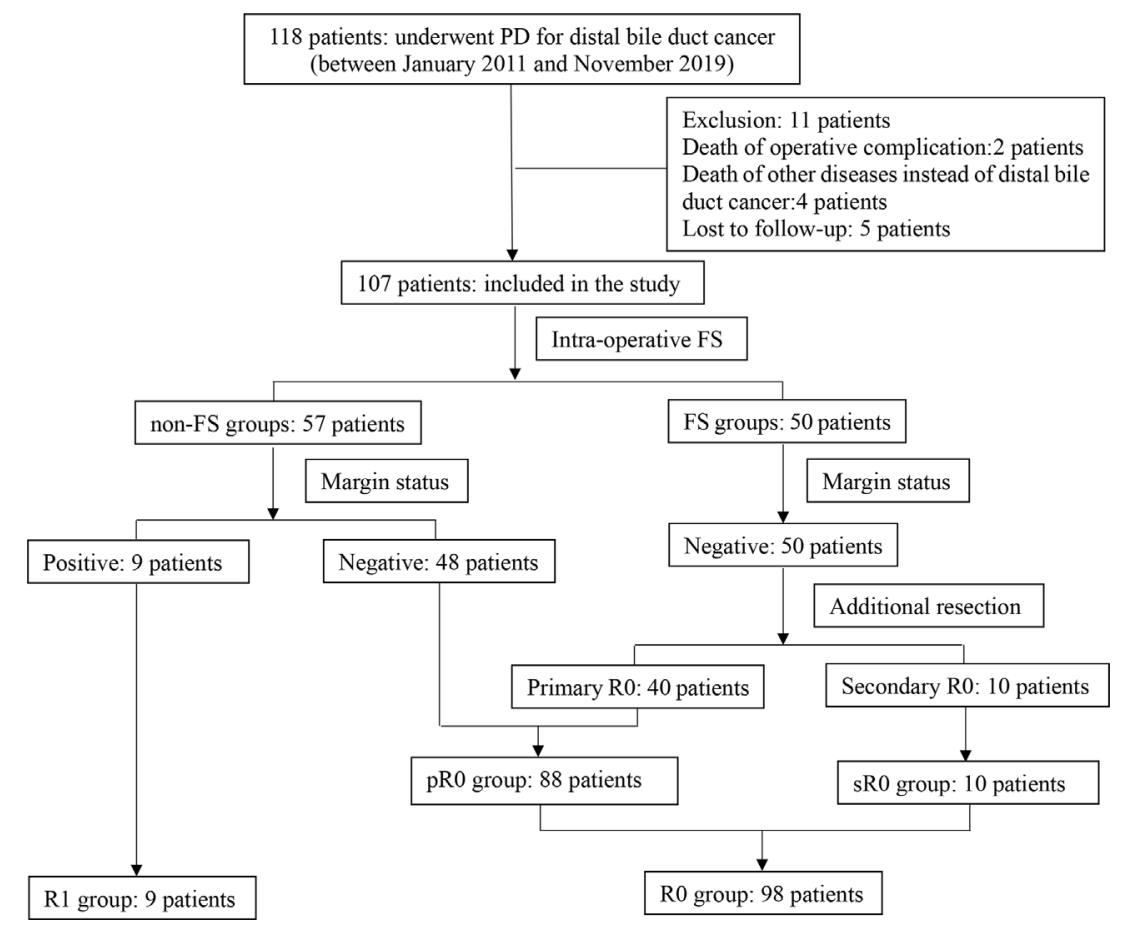

FIGURE 1 | Flow chart of patient selection and classification of the study. 107 patients were included and divided into different groups based on use of FS (FS and non-FS groups) and resection margin status (pRO, sR0 and R1 groups).

in non-FS groups higher than that of FS group ( $15.8 \%$ vs. $0 \%$, $P=0.003)$.

Patient survival of the two groups was demonstrated in Figure 2. The median survival was 28 months in FS group and 26 months in non-FS group, while the 1-, 3- and 5-year survival of FS group was $100 \%, 26.4 \%$ and $22.0 \%$, compared to $84.2 \%$, $36.6 \%$ and $15.2 \%$ in non-FS group, respectively. There was no statistical difference of overall survival between the two groups by Kaplan-Meier analysis $(P=0.314)$.

\section{Influences of Resection Margin Status on Clinical Outcomes}

In order to view if survival of patients may differ between resection margin status, we divided the patients into two groups, namely R0 group (98 patients) and R1 group (9 patients). Kaplan-Meier analysis was then used to compare survival differences between the two groups. As shown in Figure 3, the median survival was 29 months in R0 group and 22 months in R1 group, while the 1-, 3- and 5-year survival in R0 group was $92.7 \%, 33.3 \%$ and $25.0 \%$ compared to $77.8 \%, 11.1 \%$ and 0 in R1 group, which showed a better survival in R0 group of patients than in $\mathrm{R} 1$ group with a statistically significant difference $(P=0.006)$.

To assess the impact of additional resection to achieve a secondary R0 resection margin, we subdivided R0 group into pR0 (88 patients) and sR0 (10 patients) as mentioned above. Statistical analysis among pR0, sR0 and R1 groups was conducted with Kaplan-Meier curve and illustrated in
Figure 4, which showed significant difference in terms of survival among the three groups $(P=0.011)$. A more detailed analysis between subgroups was then conducted, revealing that there was significant difference between $\mathrm{pR} 0$ and R1 group $(P=0.005)$, while no statistical difference was observed concerning pR0 vs. sR0 $(P=0.211)$ and sR0 vs. R1 groups $(P=0.262)$.

\section{Multivariable Cox Regression Analysis for Risk Factors on Overall Survival}

On multivariate Cox regression analysis, independent factors associated with reduced OS were revealed to be pre-operative biliary drainage, positive lymph invasion and R1 resection margin (Table 2). It was notable that conversion of $\mathrm{R} 1$ to $\mathrm{R} 0$ (i.e. sR0) presented a similar survival with $\mathrm{pR} 0$ group, with a HR of 1.089 (95\% CI $=0.438-2.705, P=0.854)$, while $\mathrm{R} 1$ resection showed a poor survival with a HR of 3.233 (95\% CI $=1.517-$ 6.890, $P=0.002$ ).

\section{DISCUSSION}

Although the incidence rate of dCCA is decreasing and resectability is increasing with medical developments over last decades, the long-term survival of patients is still dismal $(5,6)$. In this study, there were 107 patients who underwent PD or PPPD for dCCA in our hospital during the study period, with a median survival of 27 months, 1-, 3- and 5-year survival of 91.5\%, 31.3\% 
TABLE 1 | Patient demographics and clinicopathologic characteristics.

\begin{tabular}{|c|c|c|c|c|c|c|c|c|}
\hline & \multirow{2}{*}{$\begin{array}{c}\text { Total } \\
(\mathrm{N}=107)\end{array}$} & \multicolumn{3}{|c|}{ Subgroups by FS } & \multicolumn{4}{|c|}{ Subgroups by resection margin status } \\
\hline & & $\begin{array}{l}\text { non-FS group } \\
(n=57)\end{array}$ & $\begin{array}{l}\text { FS group } \\
(n=50)\end{array}$ & $P$-value & pR0 group $(n=88)$ & sR0 group $(n=10)$ & R1 group $(n=9)$ & $P$-value \\
\hline Age (mean $\pm \mathrm{SD}$, years) & $62.3 \pm 8.21$ & $62.0 \pm 8.46$ & $62.7 \pm 7.96$ & 0.637 & $62.2 \pm 8.14$ & $60.5 \pm 9.55$ & $65.6 \pm 7.32$ & 0.388 \\
\hline Sex & & & & 0.486 & & & & 0.106 \\
\hline Male & $70(65.4 \%)$ & $39(68.4 \%)$ & $31(62.0 \%)$ & & $60(68.2 \%)$ & 7 (70.0\%) & $3(33.3 \%)$ & \\
\hline Female & 37 (34.6\%) & $18(31.6 \%)$ & 19 (38.0\%) & & $28(31.8 \%)$ & $3(30.0 \%)$ & $6(66.7 \%)$ & \\
\hline Complication & $40(37.4 \%)$ & $23(40.4 \%)$ & 17 (34.0\%) & 0.498 & $33(37.5 \%)$ & $4(40.0 \%)$ & 3 (33.3\%) & 0.955 \\
\hline CA199 (mean, U/ml) & $242.1 \pm 297.44$ & $255.0 \pm 317.86$ & $227.3 \pm 274.78$ & 0.634 & $233.9 \pm 295.41$ & $167.8 \pm 150.86$ & $404.0 \pm 400.64$ & 0.188 \\
\hline Pre-operative biliary drainage & $64(90.1 \%)$ & 37 (64.9\%) & 34 (68.0\%) & 0.736 & $58(65.9 \%)$ & $9(90.0 \%)$ & $4(44.4 \%)$ & 0.108 \\
\hline Pancreatoduodenectomy & $100(93.5 \%)$ & $53(93.0 \%)$ & 47 (94.0\%) & 0.832 & $83(94.3 \%)$ & 9 (90.0\%) & 8 (88.9\%) & 0.737 \\
\hline Clavien-Dindo classification & & & & 0.841 & & & & 0.522 \\
\hline I & 61 (57.0\%) & 31 (54.4\%) & 30 (60.0\%) & & $51(58.0 \%)$ & $5(50.0 \%)$ & $5(55.6 \%)$ & \\
\hline$\|$ & $14(13.1 \%)$ & $8(14.0 \%)$ & $6(12.0 \%)$ & & $13(14.8 \%)$ & $0(0 \%)$ & $1(11.1 \%)$ & \\
\hline III & 32 (29.9\%) & $18(31.6 \%)$ & $14(28.0 \%)$ & & $24(27.3 \%)$ & $5(50.0 \%)$ & $3(33.3 \%)$ & \\
\hline Tumor differentiation & & & & 0.299 & & & & 0.723 \\
\hline Low & 46 (43.0\%) & 22 (38.6\%) & 24 (48.0\%) & & $39(44.3 \%)$ & $4(40.0 \%)$ & $3(33.3 \%)$ & \\
\hline Moderate & $51(47.7 \%)$ & $31(54.4 \%)$ & $20(40.0 \%)$ & & $42(47.7 \%)$ & $5(50.0 \%)$ & $4(44.4 \%)$ & \\
\hline High & $10(9.3 \%)$ & 4 (7.0\%) & $6(12.0 \%)$ & & 7 (8.0\%) & $1(10.0 \%)$ & $2(22.2 \%)$ & \\
\hline Tumor size (mean, cm) & $2.0 \pm 0.66$ & $1.9 \pm 0.81$ & $2.0 \pm 0.82$ & 0.846 & $1.9 \pm 0.76$ & $2.4 \pm 1.23$ & $2.1 \pm 0.67$ & 0.182 \\
\hline T stage & & & & 0.808 & & & & 0.985 \\
\hline $\mathrm{T} 1 / 2$ & $33(30.8 \%)$ & 17 (29.8\%) & $16(32.0 \%)$ & & 27 (30.7\%) & $3(30.0 \%)$ & $3(33.3 \%)$ & \\
\hline T3 & $74(69.2 \%)$ & 40 (70.2\%) & $34(68.0 \%)$ & & $61(69.3 \%)$ & 7 (70.0\%) & $6(66.7 \%)$ & \\
\hline Pancreatic invasion & 67 (62.6\%) & 37 (64.9\%) & $30(60.0 \%)$ & 0.600 & $54(61.4 \%)$ & $6(60.0 \%)$ & 7 (77.8\%) & 0.615 \\
\hline Duodenal invasion & 17 (15.9\%) & 12 (21.1\%) & 5 (10.0\%) & 0.119 & $15(17.0 \%)$ & $1(10.0 \%)$ & $1(11.1 \%)$ & 0.778 \\
\hline Microvascular invasion & $20(18.7 \%)$ & $11(19.3 \%)$ & $9(18.0 \%)$ & 0.864 & $18(20.5 \%)$ & $1(10.0 \%)$ & $1(11.1 \%)$ & 0.601 \\
\hline Lymph invasion & 24 (22.4\%) & $12(21.1 \%)$ & 12 (24.0\%) & 0.715 & $21(23.9 \%)$ & $1(10.0 \%)$ & 2 (22.2\%) & 0.609 \\
\hline Perineural invasion & 36 (33.6\%) & $19(33.3 \%)$ & 17 (34.0\%) & 0.942 & 29 (33.0\%) & 5 (50.0\%) & $2(22.2 \%)$ & 0.418 \\
\hline Adjuvant treatment & 33 (30.8\%) & 19 (33.3\%) & $14(28.0 \%)$ & 0.551 & $23(26.1 \%)$ & $5(50.0 \%)$ & $5(55.6 \%)$ & 0.074 \\
\hline
\end{tabular}

and $21.5 \%$, respectively, which were in consistence with other reports $(7,16)$.

Surgery still remains the only potentially curative treatment for dCCA patients, and intraoperative FS is broadly used to ensure a R0 resection margin which is considered to be an important prognostic factor for long-term survival (7-9). However, the use and clinical value of FS is controversial as some pitfalls and disagreement with permanent section (PS) may present. For example, the diagnosis of some lesions (severe dysplasia, carcinoma in situ, and intraepithelial neoplasia) might be subjective due to the lack of standard diagnostic criteria $(17,18)$. Preoperative manipulation, such as biliary drainage, stenting or biopsy, might lead to inflammation, fibrosis and other reactive changes of epithelium which may influence the diagnosis of resection margin $(11,17-19)$. Besides, it was challenging even for experienced pathologists to differentiate true cytologic atypia and reactive gland from invasive carcinoma $(19,20)$. There were several studies focusing on the use of FS during PD procedure for pancreatic cancer, that showed FS did improve the rate of R0 resection by $6.0 \%$ to $8.4 \%$, but with no evidence of improved survival by extending the pancreatic resection to obtain a sR0 resection margin (21-24). But limited data is known about the use of FS to obtain a sR0 resection margin and its impact on survival of dCCA patients.

In present study, we demonstrated that intraoperative use of FS significantly increased the rate of R0 resection, but it did not improve the overall survival of patients. Nevertheless, our further analysis revealed that $\mathrm{R} 0$ resection had a significant better survival than R1 resection. Indeed, most studies agreed that R1 resection had a strong correlation with local recurrence, and complete R0 resection was a major prognostic factor for longterm survival of CCA patients (7-9). But there were some other reports showed that patients underwent $\mathrm{R} 1$ resection lived longer than expected, and it seemed that most of them had a positive ductal margin of carcinoma in situ (not invasive carcinoma) (25, 26). It needs to be mentioned that in our study, there was one patient in FS group having carcinoma in situ at the primary resection margin and was converted to sR0 by additional resection; and there was another patient in non-FS group having carcinoma in situ at the resection margin revealed by postoperative PS which was allocated to R1 group. Both patients underwent postoperative chemotherapy and survived till last follow-up (21 and 29 months). Long-term follow-up for this kind of patients should be emphasized, as they may have better survival than invasive carcinoma at resection margin.

Next, we subdivided the R0 group into pR0 and $\mathrm{sR} 0$ to assess if the use of FS on ensuring a sR0 could provide some survival benefits. Data revealed that pR0 group of patients shared the best long-term survival than R1 group, while sR0 group did not show statistical difference compared to R1 group. But it is of interest that survival of sR0 group and pR0 group did not show statistical difference either, which mean they may have similar survival. Here, we can see a statistical dilemma between these three groups (i.e. $\mathrm{pR} 0 v s$. R1, sR0 vs. R1 and sR0 vs. pR0), we think it is related to two reasons: firstly, the follow-up time was not long enough for all patients, and there were still some survivors at the time of 


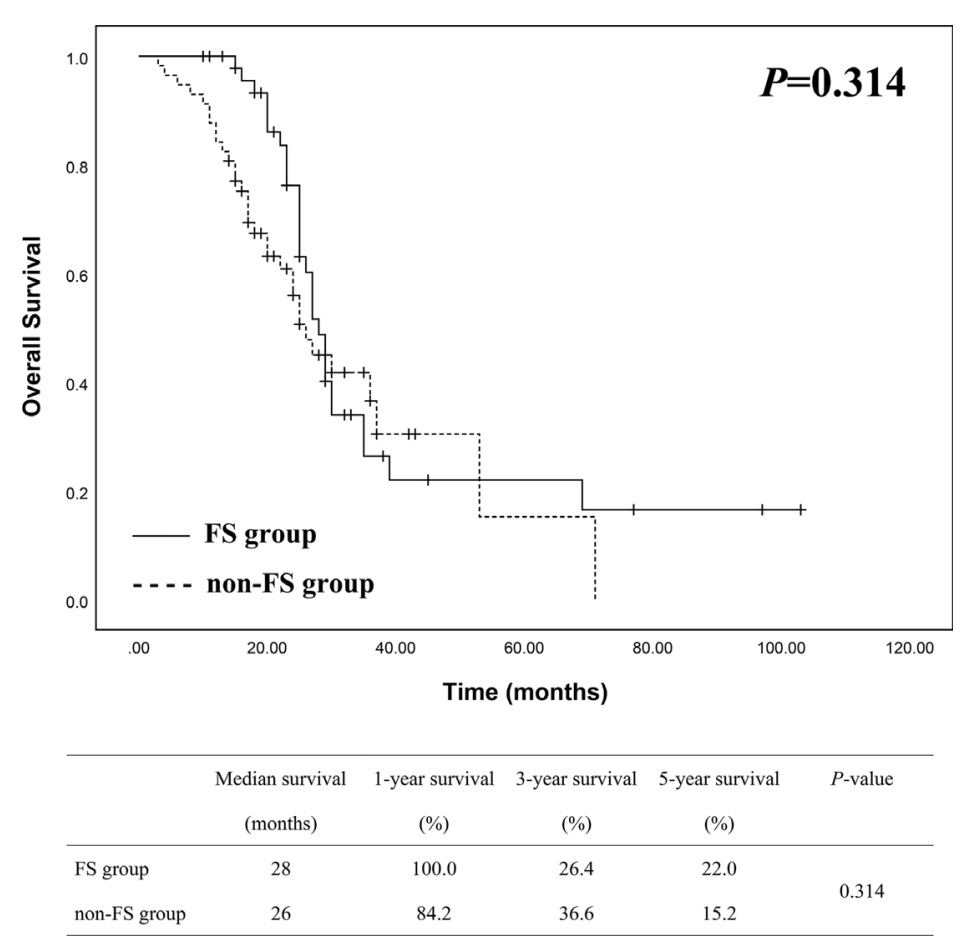

FIGURE 2 | Survival analysis between FS $(n=50)$ and non-FS $(n=57)$ groups showed no statistical difference.

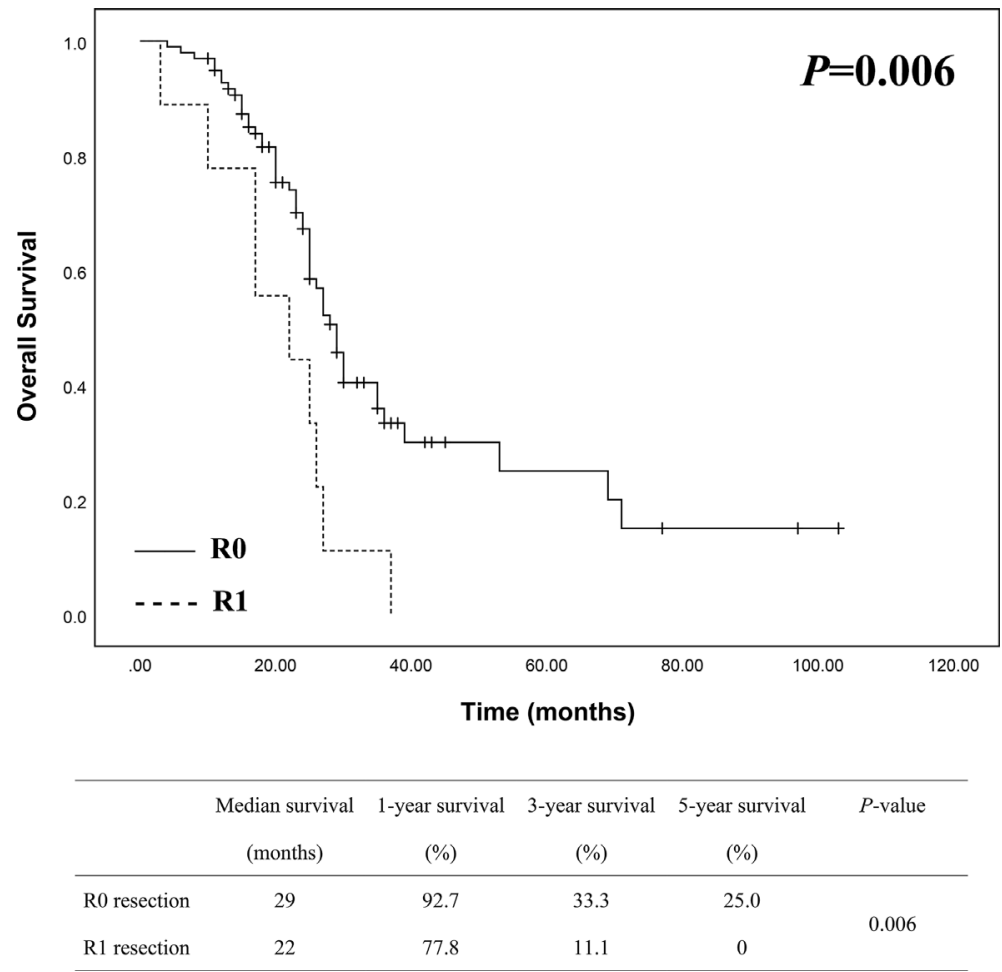

FIGURE 3 | Survival analysis between RO $(n=98)$ and R1 $(n=9)$ groups showed a better survival of R0 resection with a statistically significant difference. 


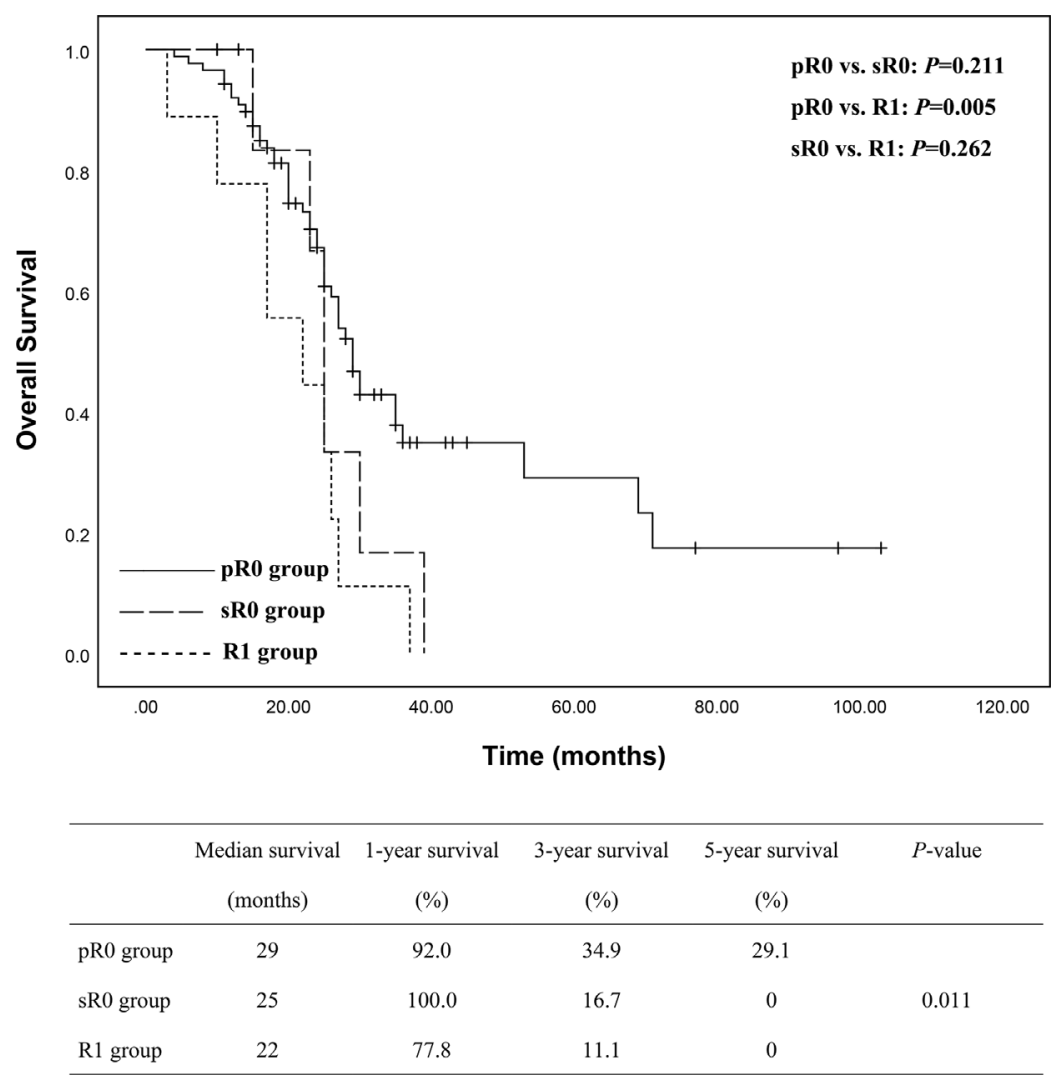

FIGURE 4 | Subgroups analyses of the overall survival between pRO $(n=88)$, sR0 $(n=10)$ and R1 $(n=9)$ groups showed a significant better survival for pR0 group of patients, but no statistical differences between sRO vs. pRO and sRO vs. R1.

last follow-up; secondly, the numbers of patients in sR0 and R1 group were small which could affect the power of statistical analyses. Thus, long-term follow-up and large number of patients should be emphasized for future studies.
Nevertheless, the present data showed that R0 resection provided a significantly better survival than R1 resection, especially for pR0 resection group. Intraoperative FS should be recommended as it could dramatically increase $\mathrm{R} 0$ resection rate

TABLE 2 | Multivariate analysis of variables associated with overall survival.

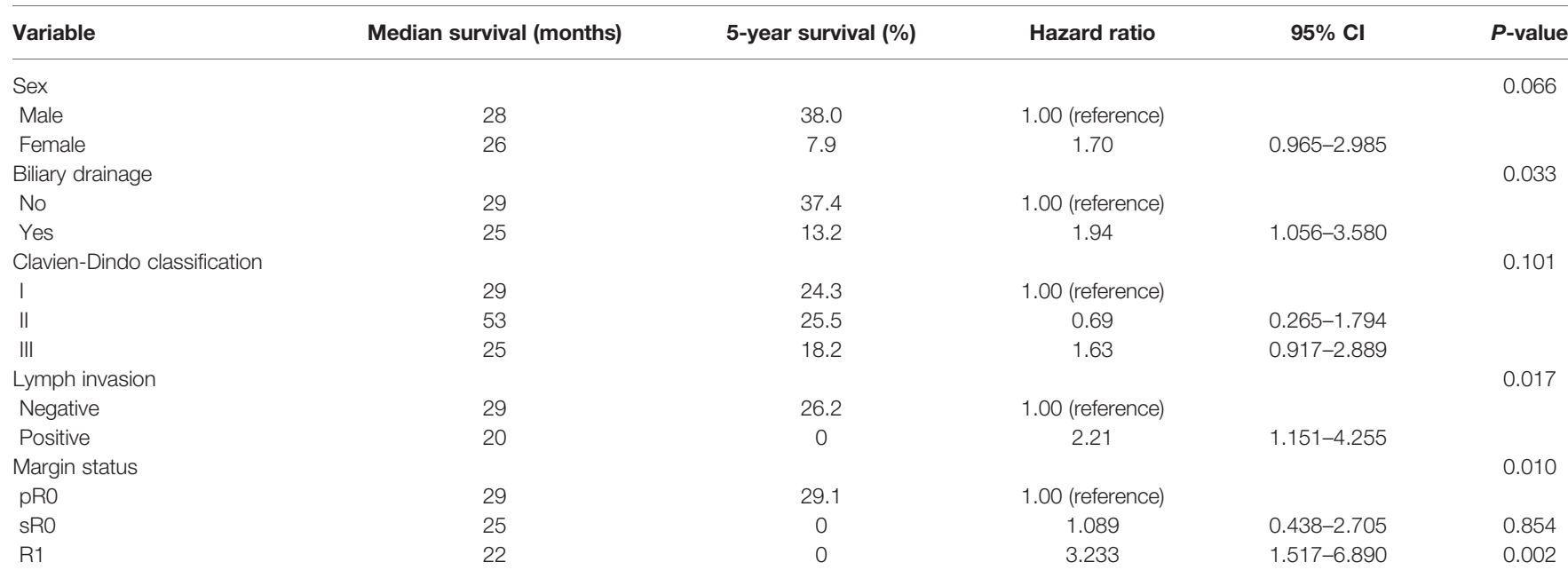


during operation, and re-resection of positive margin should be considered at the discretion of experienced surgeons as sR0 resection showed similar survival with $\mathrm{pR} 0$ resection in our study. Indeed, many studies approved additional resection to achieve sR0 resection for pCCA and eCCA (extrahepatic cholangiocarcinoma) when the primary resection margin was positive on intraoperative FS (7-9, 26-28). In a recent research, Park et al. compared overall survival between R0 on first bile duct resection (pR0) and R0 after additional resection (sR0), and supposed there was no difference in OS between the two groups (29). In a study by Tsukahara et al., they demonstrated the survival rate of seven patients achieved R0 status after additional resection (sR0) was similar to that of pR0, and suggested additional resection was beneficial to some selected patients with positive margin of carcinoma in situ (CIS) (30).

There were many series investigating prognostic factors of surgically treated dCCA patients, and the commonly identified factors were lymph node invasion, pancreatic invasion, perineural invasion, resection margin status, tumor grade, blood transfusion and adjuvant therapy $(8,10,13,29,31-33)$. Our study revealed that, besides positive resection margin, preoperative biliary drainage and lymph node invasion were both independent risk factors of poor survival of dCCA patients with surgical resection (Table 2). To be noted, pancreatic invasion, perineural invasion, tumor grade (size, differentiation and $\mathrm{T}$ stage), blood transfusion and adjuvant therapy were not associated with prognosis in our study groups. Majority of the patients received preoperative biliary drainage to alleviate jaundice, and most of them were treated with PTCD which is more favorable in our hospital. Cox regression model revealed preoperative biliary drainage to be a risk factor for poor survival, as may be explained that patients who received biliary drainage always had more severe obstructive jaundice and comorbidities (a total bilirubin $>200 \mu \mathrm{mol} / \mathrm{L}$ and/or severe comorbidities that need to take long time to be improved). A recent investigation by Miura et al. evaluated prognostic impact of the type of preoperative biliary drainage, and concluded that PTCD should be avoided since patients in their cohort who underwent PTCD had poorer overall survival and higher incidence of liver metastasis than those who underwent endoscopic biliary drainage (ENBD+ERBS) (34). As case numbers of ENBD and ERBS in our study were limited, it was inefficient to analyze survival data among subgroups. Future large volume or multicenter randomized control trials should be designed to get this issue clarified. Lymph node invasion was indicated as another independent risk factor for poor survival in our study. Although it has already reached a consensus that lymph node metastasis is a prognostic indicator by most studies $(8,10,29)$, whether to take extended lymphadenectomy is still under controversy, as some studies suggested it could not improve survival but help to stage and predict prognosis, while some other studies advocated extended lymphadenectomy for a survival advantage (35-38). Again, randomized control trials should be emphasized for future studies on this issue.

There were some limitations for this study. Firstly, this was a primary study with small sample size, especially for sR0 and R1 groups of patients, and the median follow-up period was $24.0 \pm$ 12.27 months, so the study was subject to an underpowered statistical difference. Secondly, this was a retrospective analysis with some inherent pitfalls such as participant selection bias. Thirdly, this was a single center study and therefore may not be generalizable more broadly. So large volume or multicenter randomized control trials should be designed for future investigation.

To be concluded, although intraoperative FS did not show an overall survival benefit compared to non-FS group in our study, it still should be recommended as it dramatically increased the rate of $\mathrm{R} 0$ resection, and $\mathrm{R} 0$ resection showed a better survival outcomes compared to R1 group. A pR0 resection should also be encouraged and if not, a sR0 should be considered at the discretion of surgeons as it showed similar survival with pR0 resection. Besides resection margin status, preoperative biliary drainage and lymph node metastasis were also revealed to be independent prognostic factors by multivariate analyses. And multi-center randomized control trials with large patient volume should be considered for future studies.

\section{DATA AVAILABILITY STATEMENT}

The raw data supporting the conclusions of this article will be made available by the authors, without undue reservation.

\section{ETHICS STATEMENT}

The studies involving human participants were reviewed and approved by Medical Ethics Committee of Qilu Hospital, Shandong University. The patients/participants provided their written informed consent to participate in this study.

\section{AUTHOR CONTRIBUTIONS}

Study concepts: ZC, BY, and TL. Study design: ZC, BY, and TL. Data acquisition: BY and JB. Quality control of data and algorithms: ZC, BY, and QL. Data analysis and interpretation: ZC, BY, and ZD. Statistical analysis: ZC, BY, and BX. Manuscript preparation: ZC and BY. Manuscript editing: ZC and BY. Manuscript review: ZC, XZ, and TL. All authors contributed to the article and approved the submitted version.

\section{FUNDING}

This study was supported by the Taishan Scholars Program for Young Experts of Shandong Province (tsqn20161064) and the National Natural Science Foundation of China (82073200 \& 81874178) for TL. 


\section{REFERENCES}

1. Bergquist A, von Seth E. Epidemiology of Cholangiocarcinoma. Best Pract Res Clin Gastroenterol (2015) 29(2):221-32. doi: 10.1016/j.bpg.2015. 02.003

2. Rizvi S, Gores GJ. Pathogenesis, Diagnosis, and Management of Cholangiocarcinoma. Gastroenterology (2013) 145(6):1215-29. doi: 10.1053/j.gastro.2013.10.013

3. Fritz AG. International Classification of Diseases for Oncology: ICD-O. 3rd Ed. Geneva: World Health Organization (2000). p. vii, 240.

4. Khan SA, Davidson BR, Goldin RD, Heaton N, Karani J, Pereira SP, et al. Guidelines for the Diagnosis and Treatment of Cholangiocarcinoma: an Update. Gut (2012) 61(12):1657-69. doi: 10.1136/gutjnl-2011-301748

5. Esnaola NF, Meyer JE, Karachristos A, Maranki JL, Camp ER, Denlinger CS. Evaluation and Management of Intrahepatic and Extrahepatic Cholangiocarcinoma. Cancer (2016) 122(9):1349-69. doi: 10.1002/cncr.29692

6. Lee RM, Maithel SK. Approaches and Outcomes to Distal Cholangiocarcinoma. Surg Oncol Clin N Am (2019) 28(4):631-43. doi: 10.1016/j.soc.2019.06.014

7. Chua TC, Mittal A, Arena J, Sheen A, Gill AJ, Samra JS. Resection Margin Influences Survival After Pancreatoduodenectomy for Distal Cholangiocarcinoma. Am J Surg (2017) 213(6):1072-6. doi: 10.1016/ j.amjsurg.2016.09.049

8. DeOliveira ML, Cunningham SC, Cameron JL, Kamangar F, Winter JM, Lillemoe KD, et al. Cholangiocarcinoma: Thirty-One-Year Experience With 564 Patients At a Single Institution. Ann Surg (2007) 245(5):755-62. doi: 10.1097/01.sla.0000251366.62632.d3

9. Allen PJ, Reiner AS, Gonen M, Klimstra DK, Blumgart LH, Brennan MF, et al. Extrahepatic Cholangiocarcinoma: a Comparison of Patients With Resected Proximal and Distal Lesions. HPB (Oxford) (2008) 10(5):341-6. doi: 10.1080/ 13651820802276630

10. Choi SB, Park SW, Kim KS, Choi JS, Lee WJ. The Survival Outcome and Prognostic Factors for Middle and Distal Bile Duct Cancer Following Surgical Resection. J Surg Oncol (2009) 99(6):335-42. doi: 10.1002/jso.21238

11. Konishi M, Iwasaki M, Ochiai A, Hasebe T, Ojima H, Yanagisawa A. Clinical Impact of Intraoperative Histological Examination of the Ductal Resection Margin in Extrahepatic Cholangiocarcinoma. Br J Surg (2010) 97(9):1363-8. doi: 10.1002/bjs.7122

12. Yamaguchi K, Shirahane K, Nakamura M, Su D, Konomi H, Motoyama K, et al. Frozen Section and Permanent Diagnoses of the Bile Duct Margin in Gallbladder and Bile Duct Cancer. HPB (Oxford) (2005) 7(2):135-8. doi: 10.1080/13651820510028873

13. Sakamoto Y, Kosuge T, Shimada K, Sano T, Ojima H, Yamamoto J, et al. Prognostic Factors of Surgical Resection in Middle and Distal Bile Duct Cancer: an Analysis of 55 Patients Concerning the Significance of Ductal and Radial Margins. Surgery (2005) 137(4):396-402. doi: 10.1016/j.surg.2004. 10.008

14. Dindo D, Demartines N, Clavien PA. Classification of Surgical Complications: a New Proposal With Evaluation in a Cohort of 6336 Patients and Results of a Survey. Ann Surg (2004) 240(2):205-13. doi: 10.1097/01.sla.0000133083. 54934.ae

15. Brierley J, Gospodarowicz MK, Wittekind C. TNM Classification of Malignant Tumours. Eighth edition. Chichester, West Sussex, UK; Hoboken, NJ: John Wiley \& Sons, Inc (2017).

16. Dickson PV, Behrman SW. Distal Cholangiocarcinoma. Surg Clin North Am (2014) 94(2):325-42. doi: 10.1016/j.suc.2013.12.004

17. Wakai T, Sakata J, Katada T, Hirose Y, Soma D, Prasoon P, et al. Surgical Management of Carcinoma in Situ At Ductal Resection Margins in Patients With Extrahepatic Cholangiocarcinoma. Ann Gastroenterol Surg (2018) 2:359-66. doi: 10.1002/ags3.12196

18. Konishi M, Ochiai A, Ojima H, Hasebe T, Mano M, Ohta T, et al. A New Histological Classification for Intra-Operative Histological Examination of the Ductal Resection Margin in Cholangiocarcinoma. Cancer Sci (2009) 100 (2):255-60. doi: 10.1111/j.1349-7006.2008.01043.x

19. Lechago J. Frozen Section Examination of Liver, Gallbladder, and Pancreas. Arch Pathol Lab Med (2005) 129:1610-8. doi: 10.1043/1543-2165(2005)129 [1610:FSEOLG]2.0.CO;2
20. Furukawa T, Higuchi R, Yamamoto M. Clinical Relevance of Frozen Diagnosis of Ductal Margins in Surgery of Bile Duct Cancer. J Hepatobil Pancreat Sci (2014) 21(7):459-62. doi: 10.1002/jhbp.73

21. Kooby DA, Lad NL, Squires MH,3, Maithel SK, Sarmiento JM, Staley CA, et al. Value of Intraoperative Neck Margin Analysis During Whipple for Pancreatic Adenocarcinoma: a Multicenter Analysis of 1399 Patients. Ann Surg (2014) 260(3):494-501. doi: 10.1097/SLA.0000000000000890

22. Mathur A, Ross SB, Luberice K, Kurian T, Vice M, Toomey P, et al. Margin Status Impacts Survival After Pancreaticoduodenectomy But Negative Margins Should Not be Pursued. Am Surg (2014) 80(4):353-60. doi: $10.1177 / 000313481408000416$

23. Pang TC, Wilson O, Argueta MA, Hugh TJ, Chou A, Samra JS, et al. Frozen Section of the Pancreatic Neck Margin in Pancreatoduodenectomy for Pancreatic Adenocarcinoma is of Limited Utility. Pathology (2014) 46 (3):188-92. doi: 10.1097/PAT.0000000000000072

24. Petrucciani N, Nigri G, Debs T, Giannini G, Sborlini E, Antolino L, et al. Frozen Section Analysis of the Pancreatic Margin During Pancreaticoduodenectomy for Cancer: Does Extending the Resection to Obtain a Secondary R0 Provide a Survival Benefit? Results Syst Rev Pancreatol (2016) 16(6):1037-43. doi: 10.1016/j.pan.2016.09.004

25. Jang JY, Kim SW, Park DJ, Ahn YJ, Yoon YS, Choi MG, et al. Actual Long-Term Outcome of Extrahepatic Bile Duct Cancer After Surgical Resection. Ann Surg (2005) 241(1):77-84. doi: 10.1097/01.sla.0000150166. 94732.88

26. Sasaki R, Takeda Y, Funato O, Nitta H, Kawamura H, Uesugi N, et al. Significance of Ductal Margin Status in Patients Undergoing Surgical Resection for Extrahepatic Cholangiocarcinoma. World J Surg (2007) 31 (9):1788-96. doi: 10.1007/s00268-007-9102-7

27. Otsuka S, Ebata T, Yokoyama Y, Mizuno T, Tsukahara T, Shimoyama Y, et al. Clinical Value of Additional Resection of a Margin-Positive Distal Bile Duct in Perihilar Cholangiocarcinoma. Br J Surg (2019) 106(6):774-82. doi: 10.1002/ bjs. 11125

28. Zhang XF, Squires MH,3, Bagante F, Ethun CG, Salem a, Weber SM, et al. The Impact of Intraoperative Re-Resection of a Positive Bile Duct Margin on Clinical Outcomes for Hilar Cholangiocarcinoma. Ann Surg Oncol (2018) 25 (5):1140-9. doi: 10.1245/s10434-018-6382-0

29. Park Y, Hwang DW, Kim JH, Hong SM, Jun SY, Lee JH, et al. Prognostic Comparison of the Longitudinal Margin Status in Distal Bile Duct Cancer: R0 on First Bile Duct Resection Versus R0 After Additional Resection. J Hepatobil Pancreat Sci (2019) 26(5):169-78. doi: 10.1002/jhbp.619

30. Tsukahara T, Ebata T, Shimoyama Y, Yokoyama Y, Igami T, Sugawara G, et al. Residual Carcinoma in Situ At the Ductal Stump has a Negative Survival Effect: an Analysis of Early-Stage Cholangiocarcinomas. Ann Surg (2017) 266 (1):126-32. doi: 10.1097/SLA.0000000000001944

31. Maeta T, Ebata T, Hayashi E, Kawahara T, Mizuno S, Matsumoto N, et al. Pancreatoduodenectomy With Portal Vein Resection for Distal Cholangiocarcinoma. Br J Surg (2017) 104(11):1549-57. doi: 10.1002/bjs. 10596

32. Lyu S, Li L, Zhao X, Ren Z, Cao D, He Q. Prognostic Impact of Lymph Node Parameters in Distal Cholangiocarcinoma After Pancreaticoduodenectomy. World J Surg Oncol (2020) 18(1):262. doi: 10.1186/s12957-020-02040-1

33. Kiriyama M, Ebata T, Aoba T, Kaneoka Y, Arai T, Shimizu Y, et al. Prognostic Impact of Lymph Node Metastasis in Distal Cholangiocarcinoma. Br J Surg (2015) 102(4):399-406. doi: 10.1002/bjs.9752

34. Miura F, Sano K, Wada K, Shibuya M, Ikeda Y, Takahashi K, et al. Prognostic Impact of Type of Preoperative Biliary Drainage in Patients With Distal Cholangiocarcinoma. Am J Surg (2017) 214(2):256-61. doi: 10.1016/ j.amjsurg.2017.01.010

35. Yeo CJ, Cameron JL, Sohn TA, Coleman J, Sauter PK, Hruban RH, et al. Pancreaticoduodenectomy With or Without Extended Retroperitoneal Lymphadenectomy for Periampullary Adenocarcinoma: Comparison of Morbidity and Mortality and Short-Term Outcome. Ann Surg (1999) 229 (5):613-22. doi: 10.1097/00000658-199905000-00003

36. Nguyen TC, Sohn TA, Cameron JL, Lillemoe KD, Campbell KA, Coleman J, et al. Standard vs. Radical Pancreaticoduodenectomy For Periampullary Adenocarcinoma: A Prospective, Randomized Trial Evaluating Quality of 
Life in Pancreaticoduodenectomy Survivors. J Gastrointest Surg (2003) 7 (1):1-9. doi: 10.1016/s1091-255x(02)00187-7

37. Riall TS, Cameron JL, Lillemoe KD, Campbell KA, Sauter PK, Coleman J, et al. Pancreaticoduodenectomy With or Without Distal Gastrectomy and Extended Retroperitoneal Lymphadenectomy for Periampullary Adenocarcinoma-Part 3: Update on 5-Year Survival. J Gastrointest Surg (2005) 9(9):1191-204. doi: 10.1016/j.gassur.2005.08.034

38. Kennedy EP, Yeo CJ. Pancreaticoduodenectomy With Extended Retroperitoneal Lymphadenectomy for Periampullary Adenocarcinoma. Surg Oncol Clin N Am (2007) 16(1):157-76. doi: 10.1016/j.soc.2006.10.010
Conflict of Interest: The authors declare that the research was conducted in the absence of any commercial or financial relationships that could be construed as a potential conflict of interest.

Copyright $\odot 2021$ Chen, Yu, Bai, Li, Xu, Dong, Zhi and Li. This is an open-access article distributed under the terms of the Creative Commons Attribution License (CC BY). The use, distribution or reproduction in other forums is permitted, provided the original author(s) and the copyright owner(s) are credited and that the original publication in this journal is cited, in accordance with accepted academic practice. No use, distribution or reproduction is permitted which does not comply with these terms. 